Research Article

\title{
Development of International Agricultural Trade Using Data Mining Algorithms-Based Trade Equality
}

\author{
Liu Yan (iD) \\ School of Business Administration, Zhongnan University of Economics and Law, Wuhan 430073, China \\ Correspondence should be addressed to Liu Yan; liuyan@stu.zuel.edu.cn
}

Received 26 May 2021; Accepted 22 June 2021; Published 2 July 2021

Academic Editor: Fazlullah Khan

Copyright (C) 2021 Liu Yan. This is an open access article distributed under the Creative Commons Attribution License, which permits unrestricted use, distribution, and reproduction in any medium, provided the original work is properly cited.

The development of international agriculture trade during the COVID-19 pandemic has encountered significant challenges. The processing of international agricultural trade data using machine learning techniques needs to be improved to perform effective analysis of agricultural trade. An essential issue for international agricultural trade is the accurate yield estimation for the numerous crops involved in international trade. Data mining techniques are the necessary approach for accomplishing practical and effective solutions for this problem. This paper combined the bidirectional encoder representations from transformers (BERT) model to conduct data mining and developed a trade data analysis system with efficient data analysis capabilities. Our results indicate that our model does reasonably well and obtains adequate information in deciding international agricultural trade. It can also be instrumental for policy and decision-making regarding international agricultural trade.

\section{Introduction}

The COVID-19 pandemic has completely disrupted the world economy. The world economy is facing a reshuffle situation; in particular, the agricultural economy is also experiencing more significant fluctuations and challenges. Agricultural trade is related to a country's economy and closely related to the needs of the people worldwide. China is also undergoing a structural transformation of the agricultural economy. The development of international agricultural trade is of great significance to the rise of China's overall economy [1]. The growth of international agricultural trade requires Chinese agricultural products to have strong international market competitiveness. The effective means to enhance competitiveness is to frame effective plans through data analysis, increase the market share of Chinese agricultural products, and effectively improve the market development ability of Chinese agricultural products in international trade. To improve China's international agricultural trade competitiveness, it is necessary to use the agricultural land [2] rationally. At the same time, we need to combine the actual needs of the international people and the economic benefits of agricultural products, China's climate, and land characteristics to choose crops with the best economic benefits and a specific yield. Likewise, it is also necessary to fully understand competitors' agricultural strength and products, seizing market opportunities, as well as expanding sales channels for agricultural products. Moreover, it is also required to focus on relevant international trade policies of various countries. In particular, forcibly interfering in normal economic trade through political means in some countries will directly lead to large fluctuations in international agricultural trade [3].

When formulating international agricultural trade development plans, various factors must be effectively analyzed to provide an excellent theoretical basis for the development of international trade. However, the major problem in the analysis of international trade is that the factors are uncontrollable. Moreover, it is challenging to discover the influential factors from the massive international agricultural trade data to establish the foundation for the subsequent development of international agricultural trade. Therefore, it is essential to develop a scientific and effective data mining system [4].

The data mining process provides a framework to extract nontrivial information from data. With the advent of 
massive storage, advanced data collection, and computing paradigms, the data at our disposal are only increasing. To extract information from these huge data assets, we need to use advanced approaches like data mining algorithms, in addition to standard statistical processing [5]. Although many of these algorithms can extract valuable information, it is up to the data mining professional to skillfully employ the suitable algorithms and transform a business problem into a data problem. Like any other technology, data mining provides options in terms of algorithms and parameters within the algorithms. Therefore, this study focuses on developing international agricultural trade in combination with the data mining model to improve the effectiveness of data mining as much as possible and overcome the existing problems [6]. The major contributions of this work are as follows:

(1) This work combines the BERT model to conduct international agricultural trade data mining and analyzes the current situation of China's agricultural international trade

(2) It analyzes the future situation of China's agricultural international trade and explores the future development direction of international agricultural trade

The remainder of the paper is structured as follows. Section 2 describes the related work. Section 3 illustrates the proposed data mining model. In Section 4, different results are given for model evaluation. Finally, the conclusion is given in Section 5.

\section{Related Work}

Agricultural trade plays a significant role in the sustainability of global and regional food systems. Trade among countries allows food to shift from surplus to deficit areas. It helps to increase the variety of products on offer. Without trade, the pressure on local and national food systems would be much greater and create significant burdens on natural resources and government budgets. The role of data mining in developing international agriculture trade is a leading nextgeneration agricultural intelligence [7]. This enables land users to make better decisions by alerting them to hidden patterns in discovered data. Statistics and machine learning techniques are gaining popularity in the analysis of international agriculture trade data. There are different methodologies developed and evaluated by researchers worldwide in the field of agriculture trade data analysis. Ramesh and Vardhan [8] analyzed the agriculture data for the years 1965-2009. Rainfall data were categorized into four clusters by adopting the K-means clustering method. The purpose of this work is to find suitable data models that achieve high accuracy and a high generality in terms of international agricultural yield prediction capabilities. Rahman Motiur et al. [9] studied the impact of climate conditions (rainfall and temperature) on agriculture products. The entire dataset was divided into 3-month phases (March to June, July to October, and November to February). In preprocessing agricultural data, the average for every attribute was taken. Clustering was performed to find the sharable group of a region based on a similar weather attribute. The authors in [10] studied the effects of soil characteristics on the production of agricultural products using data mining. K-means clustering was used for clustering soils in combination with geographical position system (GPS) based technologies. The authors in [10] have done a wide study on the predictive ability of machine learning algorithms such as artificial neural networks, multiple linear regression, regression trees, support vector regression, and k-nearest neighbour on international agricultural crop yield production [11]. Alberto et al. [12] predicted the variation in wheat yield based on online multilayer soil data and satellite imagery crop growth characteristics. International agricultural trade data is massive, including satellite images that also need lots of cleansing to derail the targets [13]. This study performs data analysis of agricultural data using the BERT model to improve China's international agricultural trade development. It constructs a trade data analysis system with data analysis capabilities.

\section{Data Mining Model}

In this paper, data mining is implemented through the BERT model. BERT is an open-source machine learning framework. BERT stands for bidirectional encoder representations from transformers. It is a deep machine learning model with multiple layers in which every output element is linked to every input element, and the weightings between them are dynamically computed based upon their connection $[14,15]$. The general BERT transformation is shown in Figure 1.

The output of each sublayer is expressed as

$$
\text { Sublayer_output }=\operatorname{norm}(x+(\operatorname{sublayer}(x))) .
$$

The position vector can be calculated by the following equation:

$$
\begin{array}{r}
\mathrm{PE}(\text { pos, } 2 i)=\sin \left(\frac{\text { pos }}{1000^{2 i / d_{\text {model }}}}\right), \\
\mathrm{PE}(\operatorname{pos}, 2 i+1)=\cos \left(\frac{\text { pos }}{1000^{2 i / d_{\text {model }}}}\right),
\end{array}
$$

where pos represents the specific position of the keyword in the massive data in the agricultural international trade data and $i$ is the direction vector. In data processing, to avoid data saturation, the data needs to be normalized, which can be expressed as

$$
\operatorname{LN}\left(x_{i}\right)=\alpha \times \frac{x_{i}-v_{L}}{\sqrt{\sigma^{2}+\varepsilon}}+\beta .
$$

The decoder layer does not input the entire agricultural trade data into the semantic vector $C$ according to a fixed length. However, it calculates $c(i)$ based on the continuously imported new agricultural trade information. Therefore, $C$ shows the real-time change. In this way, the new agricultural trade information loss problem is solved. This process can be expressed as a three-dimensional form, as shown in Figure 2.

The initial value $Z_{0}$ is introduced, and it is matched with $h_{1}$ to generate $a_{0}^{1}$. There are many match methods, including 


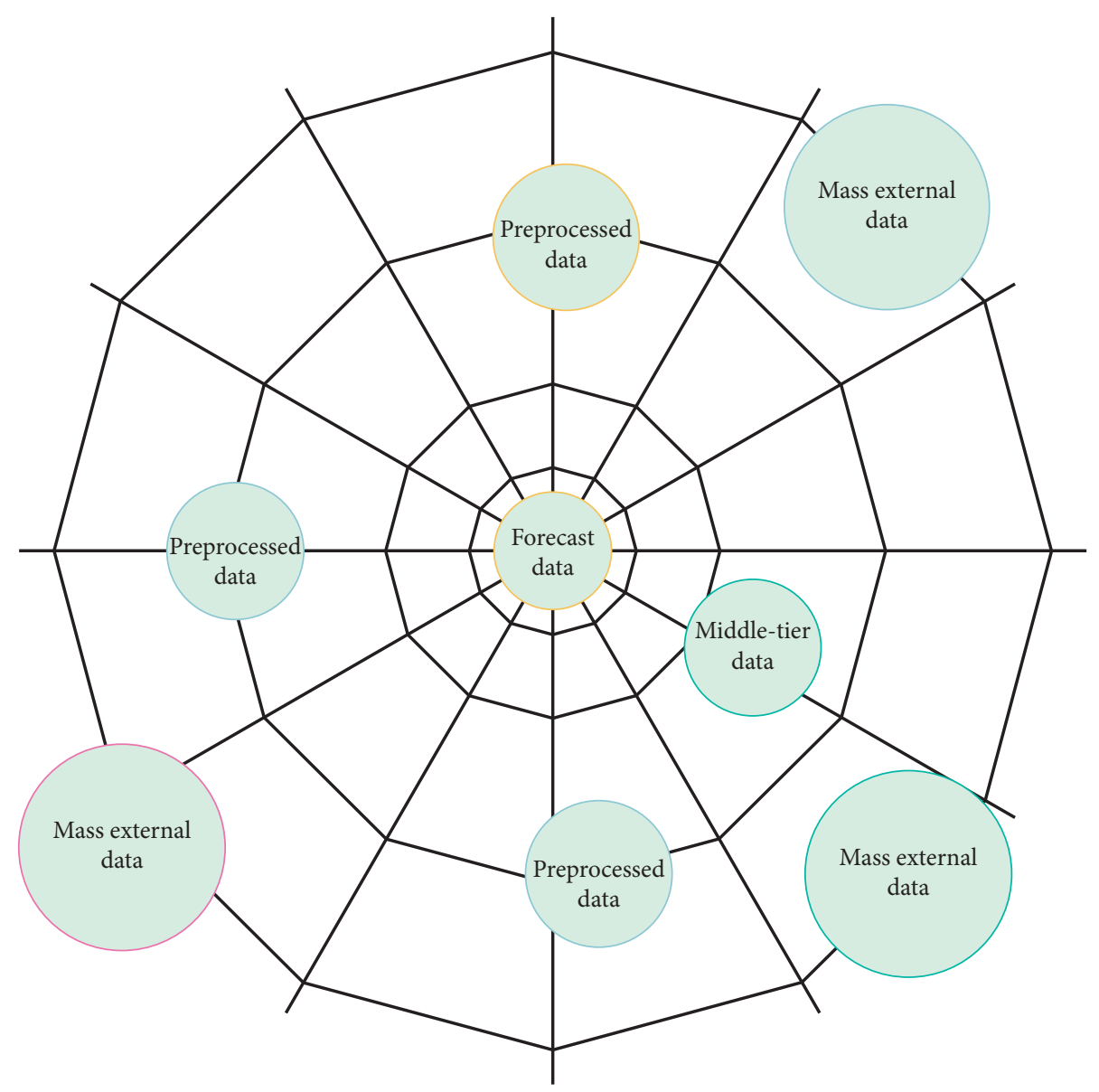

Figure 1: BERT transformation.

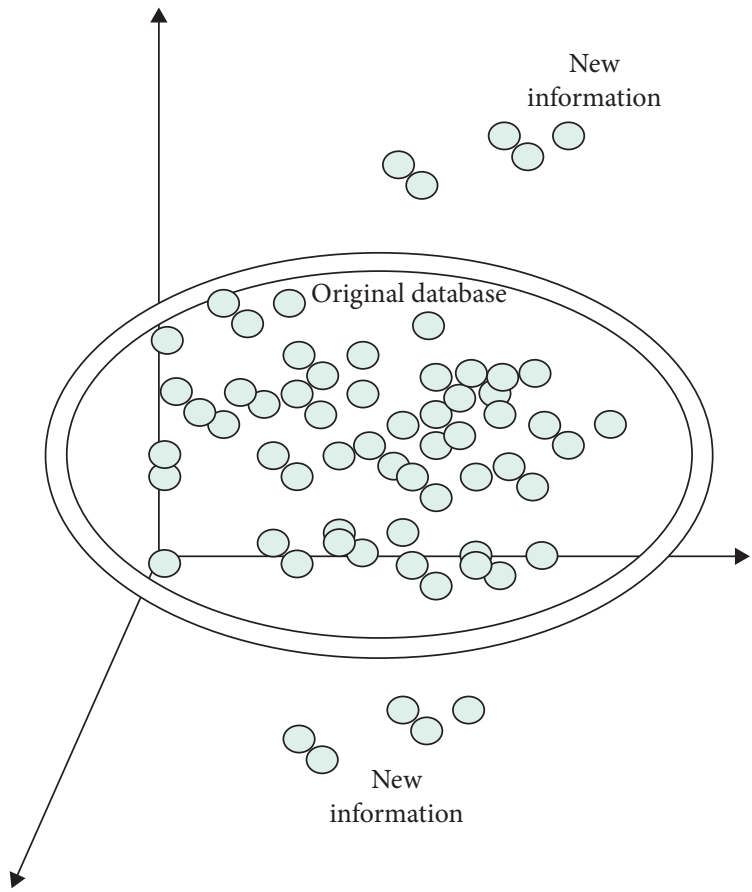

(a)

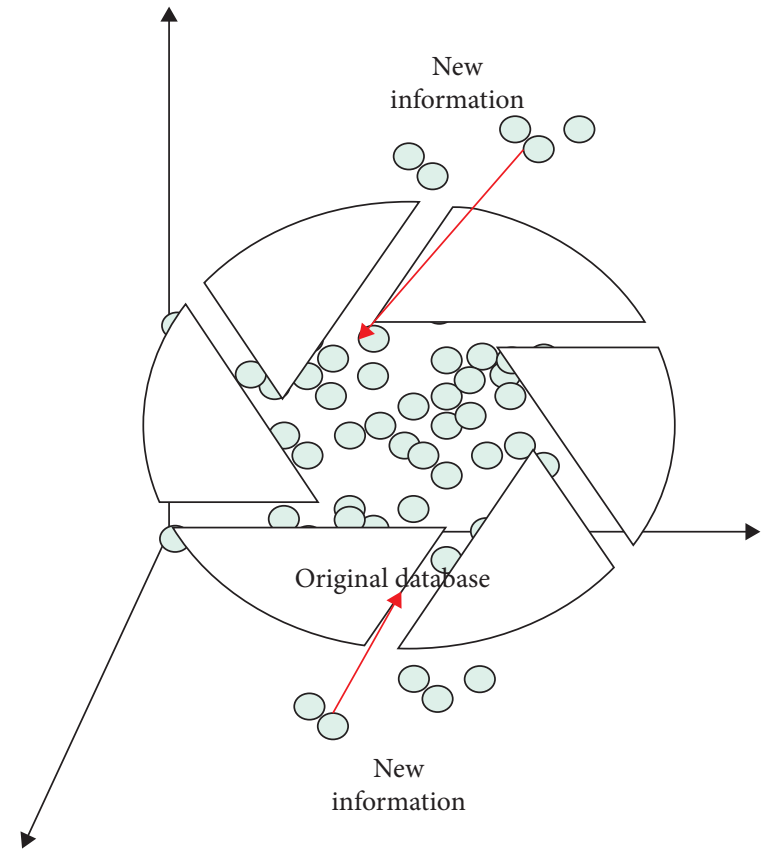

(b)

Figure 2: Attention mechanism and match operation in BERT model. (a) Attention mechanism. (b) Match operation. 
calculating the COS value of $h_{1}$ and $Z_{0}$. $Z_{0}$ sequentially performs a match operation with $h_{i}$ to calculate and obtain $a_{0}^{i} . a_{0}^{i}$ is subjected to softmax processing, and a weight is added to each $a_{0}^{i}$. Then, the softmax-processed $a_{0}^{i}$ is summed to obtain $c_{0}$. Because of the different weights, the probability of $c_{0}$ at this time tends to be closer to $h_{1}$ and $h_{2}$. At this time, $c_{0}$ is used as the input of the decoder layer to perform decoder layer operations with $z_{0}$ and generate $z_{1}$. The generated $z_{1}$ is again used as the initial value of the encoder to perform a match operation with each $h_{1}$. Then, $c_{1}$ will be obtained. In the same way, $c_{1}$ and the output result $y_{1}$ are taken by the decoder operation to get $z_{2}$, and then the algorithm iterates in turn. Finally, the algorithm ends when it encounters the EOS termination signal. The process can be represented as given in Figure 3.

The specific calculation of encoder is

$$
\begin{aligned}
h_{i} & =\tanh \left(W\left[h_{i-1}, x_{i}\right]+b\right), \\
o_{i} & =\operatorname{soft} \max \left(v h_{i}+c\right) .
\end{aligned}
$$

The semantic vector $C_{t}$ is computed as

$$
\begin{aligned}
z_{t} & =\tanh \left(W\left[z_{t-1}, y_{t-1}\right]\right), \\
e t_{i} & =z_{t}^{T} W h_{i}, \\
a_{t i} & =\frac{\exp \left(e_{t i}\right)}{\sum_{k=1}^{T} \exp \left(e_{t k}\right)}, \\
c_{t} & =\sum_{i=1}^{T} a_{t i} h_{i} .
\end{aligned}
$$

In the above equation, $c_{t}$ is the semantic vector generated at time $t$, and $e_{t i}$ is the degree of influence of the hidden layer state $h_{i}$ at time $i$ in the encoder layer on the hidden layer state $z_{t}$ at time $t$ decoder layer. $e_{t i}$ is normalized with probability to $a_{t i}$. The formula used is as follows:

$$
\begin{aligned}
\widetilde{z} & =\tanh \left(W\left[z_{t}, c_{t}\right]\right), \\
o_{i} & =\operatorname{soft} \max \left(v ! z_{t}+c\right) .
\end{aligned}
$$

First, the hidden layer state $z_{t}$ is calculated, and then the attention layer $\widehat{z}_{t}$ is calculated; finally, the prediction result is obtained. The self-attention mechanism can be expressed using the following equation:

$$
\operatorname{Attention}(q, v, k)=\operatorname{soft} \max \left(\frac{q k^{T}}{\sqrt{d}}\right) v .
$$

We have

$$
\begin{aligned}
q^{i} & =W^{q} a^{i}, \\
k^{i} & =W^{k} a^{i}, \\
v^{i} & =W^{v} a^{i} .
\end{aligned}
$$

After $q^{i}, k^{i}$, and $v^{i}$ are obtained, $q^{1}$ is multiplied by $k^{i}$ to get $a_{1,1}, a_{1,2}, a_{1,3}$, and $a_{1,4}$. Likewise, $q^{2}$ is multiplied by $k^{i}$ to get $a_{2,1}, a_{2,2}, a_{2,3}$, and $a_{2,4}$. By analogy, the $a_{i, j}$ matrix can be

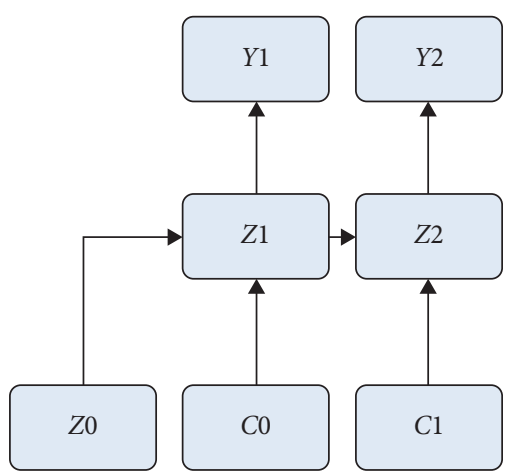

Figure 3: Decoding layer calculation.

obtained. Then, this matrix is subjected to softmax processing to obtain matrix $\widehat{a}_{i, j}$. Using $b_{1}=\sum \widehat{a}_{1, i} V^{i}, b_{1}$ can be obtained. By analogy, we get $b_{2}, b_{3}$, and $b_{4}$. The tanh function is mainly used for binary classification tasks:

$$
\tanh (x)=\frac{\sinh (x)}{\cosh (x)}=\frac{e^{x}-e^{-x}}{e^{x}+e^{-x}} .
$$

The softmax function maps a $k$-dimensional real-value vector $\left(a_{1}, a_{2}, a_{3}, a_{4}, L\right)$ to $\left(b_{1}, b_{2}, b_{3}, b_{4}, L\right)$. Among them, $b_{i}$ is a constant value between 0 and 1 . According to the size of $b_{i}$, the purpose of multiclassification is achieved. The formula is

$$
\sigma\left(z_{j}\right)=\frac{e_{z_{j}}}{\sum_{k=1}^{k} e^{z_{k}}} .
$$

The GELU function is as follows:

$$
\operatorname{GELU}(X)=0.5 X\left(1+\tanh \left(\sqrt{2} \div \pi\left(x+0.44715 x^{3}\right)\right)\right) \text {. }
$$

Using the same sequence of calculations, $a^{i}$ is divided into matrices $q^{i}, k^{i}$, and $v^{i}$. At the same time, $q^{i}$ is divided into $q^{i, 1}$ and $q^{i, 2}, k^{i}$ is divided into $k^{i, 1}$ and $k^{i, 2}$, and $v^{i}$ is divided into $v^{i, 1}$ and $v^{i, 2}$.

$$
\begin{aligned}
& q^{i, 1}=W^{q, 1} * q^{i} ; \\
& q^{i, 2}=W^{q, 2} * q^{i}, \\
& k^{i, 1}=W^{k, 1} * k^{i} ; \\
& k^{i, 2}=W^{k, 2} * k^{i}, \\
& v^{i, 1}=W^{v, 1} * v^{i} ; \\
& v^{i, 2}=W^{v, 2} * v^{i} .
\end{aligned}
$$

After obtaining $q^{i}, k^{i}$, and $v^{i}, q^{i, 1}$ is multiplied with $k^{i, 1}$ to get $a^{i, 1}$. In the same way, $q^{i, 2}$ is multiplied by $k^{i, 2}$ to get $a^{i, 2}$. Simultaneously, softmax processing is performed on $a^{i, j}$ to obtain $\widehat{a}^{i, j}$ matrix. According to $b_{1}=\sum \hat{a}^{i, j} V^{i, 1}, b^{i, 1}$ and $b^{i, 2}$ can be obtained in the same way. Similarly, $b^{i, 1}$ and $b^{i, 2}$ are multiplied by weight $W^{0}$, to obtain $b^{i}$.

\section{Model Evaluation}

This work has analyzed the massive international agricultural trade data, obtained effective information, and 


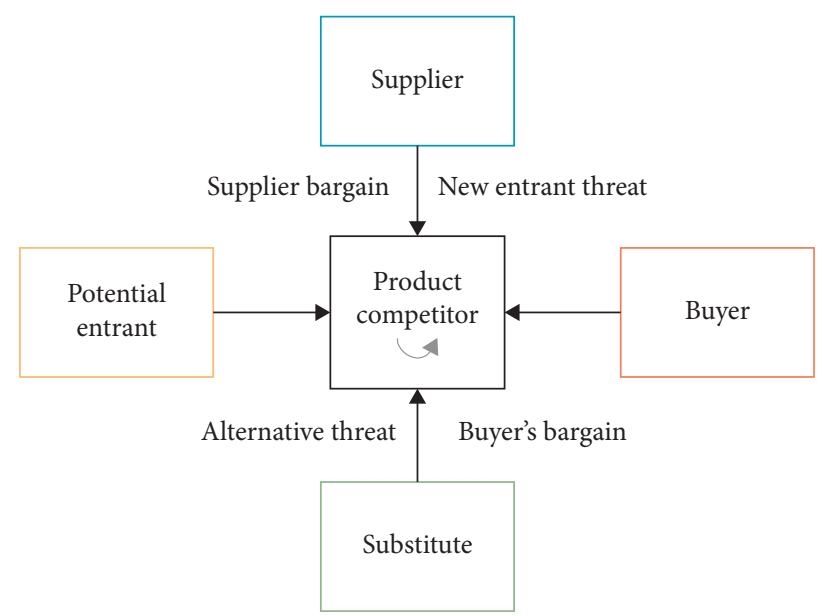

Figure 4: The Porter analysis model.

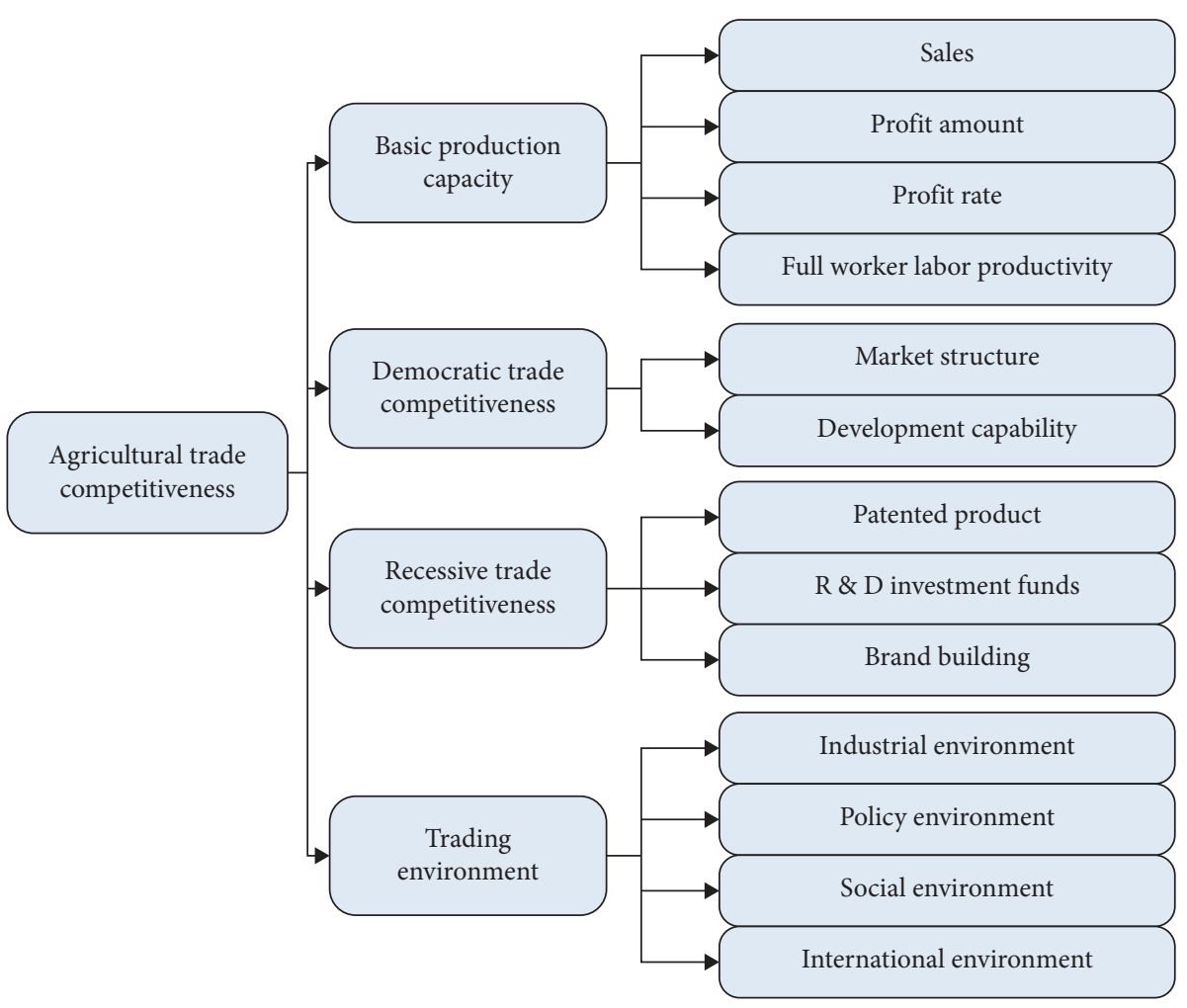

Figure 5: Structure diagram of the AHP model.

predicted effective laws [16]. For data mining, we used the competitiveness theory model, which is based on the Porter model. Porter's model identifies and analyzes five competitive forces that shape every industry and helps determine an industry's weaknesses and strengths [17]. In the present study, the five competitive forces analyzed were as follows: suppliers, buyers, potential entrants, substitutes, and product competitors. The Porter analysis model is shown in Figure 4.

Based on Porter's analysis model, the analytical hierarchy process (AHP) model was constructed. The functional structure diagram of the AHP model is shown in Figure 5.
To conduct a multifactor analysis of the international agricultural trade data, we employed the AHP model. All the factors were processed hierarchically, which is similar to the decision tree structure. The AHP is a method for organizing and analyzing complex decisions. AHP provides a rational framework for a needed decision by quantifying its criteria and alternative options and relating those elements to the overall goal. AHP is unique because it can quantify criteria and alternatives [18]. The construction of a model is generally followed by model verification. The objective of model verification was to ensure that the implementation of the model is correct. We obtained a large amount of agricultural 
TABLE 1: Statistical table of the evaluation of data mining ability.

\begin{tabular}{|c|c|c|c|c|c|c|c|}
\hline No. & Data mining & No. & Data mining & No. & Data mining & No. & Data mining \\
\hline 1 & 89.23 & 21 & 87.75 & 41 & 92.41 & 61 & 88.57 \\
\hline 2 & 88.10 & 22 & 90.58 & 42 & 90.80 & 62 & 87.60 \\
\hline 3 & 92.13 & 23 & 90.71 & 43 & 92.24 & 63 & 87.54 \\
\hline 4 & 93.06 & 24 & 92.86 & 44 & 88.86 & 64 & 91.38 \\
\hline 5 & 92.40 & 25 & 90.73 & 45 & 89.65 & 65 & 88.94 \\
\hline 6 & 91.66 & 26 & 90.52 & 46 & 92.98 & 66 & 87.46 \\
\hline 7 & 90.17 & 27 & 93.54 & 47 & 92.48 & 67 & 91.60 \\
\hline 8 & 93.26 & 28 & 89.25 & 48 & 92.03 & 68 & 88.81 \\
\hline 9 & 91.67 & 29 & 87.50 & 49 & 89.36 & 69 & 90.23 \\
\hline 10 & 87.98 & 30 & 90.01 & 50 & 89.31 & 70 & 89.70 \\
\hline 11 & 92.25 & 31 & 90.63 & 51 & 93.21 & 71 & 91.90 \\
\hline 12 & 93.27 & 32 & 91.31 & 52 & 90.21 & 72 & 93.83 \\
\hline 13 & 87.94 & 33 & 90.03 & 53 & 88.21 & 73 & 92.24 \\
\hline 14 & 89.27 & 34 & 90.76 & 54 & 90.00 & 74 & 87.73 \\
\hline 15 & 92.09 & 35 & 91.70 & 55 & 87.08 & 75 & 91.91 \\
\hline 16 & 90.38 & 36 & 89.17 & 56 & 89.79 & 76 & 93.55 \\
\hline 17 & 93.44 & 37 & 92.93 & 57 & 89.98 & 77 & 92.26 \\
\hline 18 & 89.12 & 38 & 89.88 & 58 & 89.53 & 78 & 89.27 \\
\hline 19 & 87.77 & 39 & 91.76 & 59 & 93.32 & 79 & 90.56 \\
\hline 20 & 90.05 & 40 & 90.12 & 60 & 92.76 & 80 & 91.65 \\
\hline
\end{tabular}

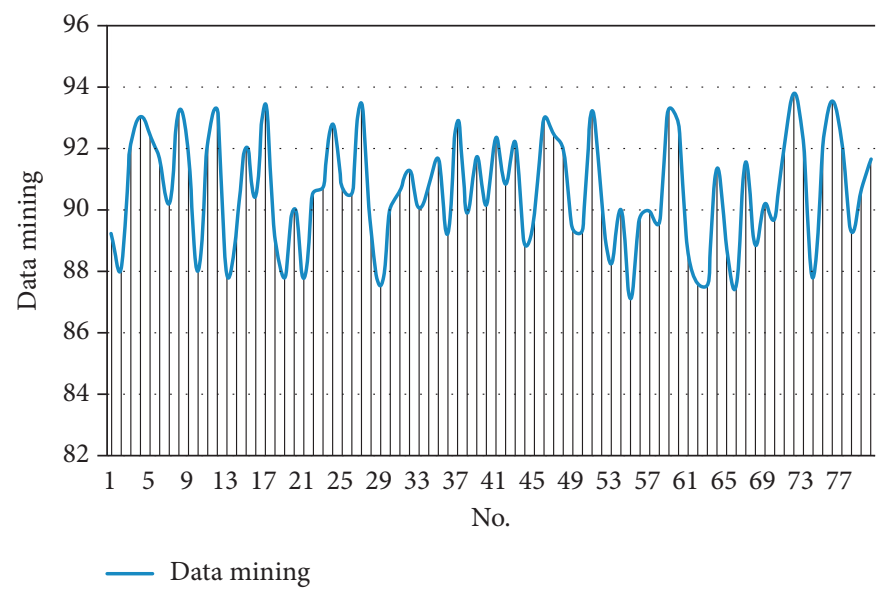

Figure 6: Statistical diagram of the evaluation of data mining ability.

international trade information from the Internet through web crawler technology to verify the model.

4.1. Data Mining Ability. Data mining is a process of discovering patterns in massive datasets involving methods at the intersection of learning [19-22]. To evaluate the data mining ability of the proposed model, we randomly extracted 80 sets of data and applied the proposed data mining technique. The results are shown in Table 1 and Figure 6, respectively. All the resultant values obtained are greater than $80 \%$, proving that the proposed data mining model can perform better decision-making and perform data mining on the international agricultural data more effectively and efficiently.

4.2. Data Analysis Capability. The pinnacle of analysis capability is applying advanced analytics to discover deep insights, make predictions, and generate recommendations. To check the data analysis capabilities of the proposed data mining model, we employed the Porter model. In addition, 80 sets of valid data were also randomly selected for the present study. Table 2 and Figure 7 show the evaluation results of data analysis capability for the proposed model. The proposed model has greater potential and good effect (data analysis $>70$ ) to analyze the international agriculture trade more efficiently.

4.3. Decision-Making Ability. Decision-making ability shows the proficiency of a system in choosing between two or more alternatives. We evaluated the proposed model's decision-making ability, compared the decision-making suggestions output with the actual situation, and used a scoring method. The obtained results for all the 80 datasets are greater than 70 and are reported in Table 3 and Figure 8, respectively. 
TABLE 2: Statistical table of the evaluation of data analysis capabilities.

\begin{tabular}{|c|c|c|c|c|c|c|c|}
\hline No. & Data analysis & No. & Data analysis & No. & Data analysis & No. & Data analysis \\
\hline 1 & 76.16 & 21 & 80.35 & 41 & 76.78 & 61 & 77.17 \\
\hline 2 & 85.43 & 22 & 76.83 & 42 & 82.28 & 62 & 76.89 \\
\hline 3 & 83.52 & 23 & 80.62 & 43 & 76.34 & 63 & 80.11 \\
\hline 4 & 79.81 & 24 & 85.37 & 44 & 78.37 & 64 & 83.82 \\
\hline 5 & 76.80 & 25 & 81.59 & 45 & 78.90 & 65 & 82.29 \\
\hline 6 & 83.17 & 26 & 84.35 & 46 & 82.16 & 66 & 76.39 \\
\hline 7 & 86.87 & 27 & 82.06 & 47 & 82.16 & 67 & 82.10 \\
\hline 8 & 86.80 & 28 & 79.85 & 48 & 83.43 & 68 & 86.18 \\
\hline 9 & 76.46 & 29 & 79.59 & 49 & 78.09 & 69 & 81.37 \\
\hline 10 & 86.66 & 30 & 83.40 & 50 & 84.51 & 70 & 78.64 \\
\hline 11 & 80.55 & 31 & 82.39 & 51 & 84.10 & 71 & 80.46 \\
\hline 12 & 85.63 & 32 & 83.10 & 52 & 79.45 & 72 & 82.89 \\
\hline 13 & 85.15 & 33 & 77.57 & 53 & 79.08 & 73 & 79.91 \\
\hline 14 & 76.92 & 34 & 83.35 & 54 & 83.80 & 74 & 77.37 \\
\hline 15 & 82.12 & 35 & 77.84 & 55 & 80.96 & 75 & 79.22 \\
\hline 16 & 80.09 & 36 & 79.15 & 56 & 76.96 & 76 & 84.73 \\
\hline 17 & 80.46 & 37 & 84.99 & 57 & 81.47 & 77 & 80.94 \\
\hline 18 & 77.08 & 38 & 86.21 & 58 & 81.09 & 78 & 77.26 \\
\hline 19 & 78.76 & 39 & 76.22 & 59 & 84.07 & 79 & 83.81 \\
\hline 20 & 83.62 & 40 & 76.84 & 60 & 79.30 & 80 & 85.19 \\
\hline
\end{tabular}

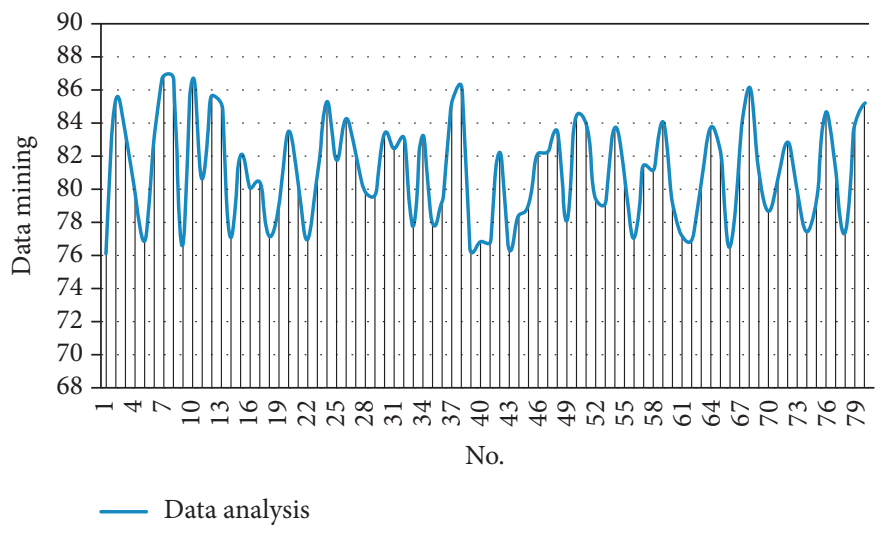

Figure 7: Statistical diagram of the evaluation of data analysis capabilities.

TABLE 3: Statistical table of the evaluation of system decision-making ability.

\begin{tabular}{|c|c|c|c|c|c|c|c|}
\hline No. & Decision effect & No. & Decision effect & No. & Decision effect & No. & Decision effect \\
\hline 1 & 79.45 & 21 & 72.00 & 41 & 78.96 & 61 & 74.08 \\
\hline 2 & 80.74 & 22 & 77.76 & 42 & 72.52 & 62 & 71.02 \\
\hline 3 & 79.46 & 23 & 80.00 & 43 & 78.52 & 63 & 74.30 \\
\hline 4 & 69.82 & 24 & 77.95 & 44 & 72.24 & 64 & 76.14 \\
\hline 5 & 75.57 & 25 & 79.31 & 45 & 72.75 & 65 & 72.13 \\
\hline 6 & 79.04 & 26 & 69.99 & 46 & 77.71 & 66 & 73.29 \\
\hline 7 & 72.26 & 27 & 70.91 & 47 & 79.42 & 67 & 72.23 \\
\hline 8 & 76.36 & 28 & 74.53 & 48 & 78.69 & 68 & 76.89 \\
\hline 9 & 70.05 & 29 & 73.56 & 49 & 72.87 & 69 & 71.55 \\
\hline 10 & 69.86 & 30 & 73.27 & 50 & 78.29 & 70 & 70.18 \\
\hline 11 & 70.54 & 31 & 70.80 & 51 & 75.08 & 71 & 79.27 \\
\hline 12 & 69.43 & 32 & 73.07 & 52 & 72.88 & 72 & 79.09 \\
\hline 13 & 75.10 & 33 & 69.81 & 53 & 74.46 & 73 & 73.12 \\
\hline 14 & 69.23 & 34 & 70.13 & 54 & 72.18 & 74 & 75.05 \\
\hline 15 & 78.48 & 35 & 73.16 & 55 & 73.01 & 75 & 80.74 \\
\hline 16 & 79.82 & 36 & 76.66 & 56 & 69.83 & 76 & 69.50 \\
\hline 17 & 72.28 & 37 & 79.89 & 57 & 73.01 & 77 & 80.98 \\
\hline 18 & 77.39 & 38 & 76.94 & 58 & 72.76 & 78 & 79.74 \\
\hline 19 & 72.36 & 39 & 78.94 & 59 & 69.48 & 79 & 70.92 \\
\hline 20 & 70.91 & 40 & 73.23 & 60 & 78.75 & 80 & 79.42 \\
\hline
\end{tabular}




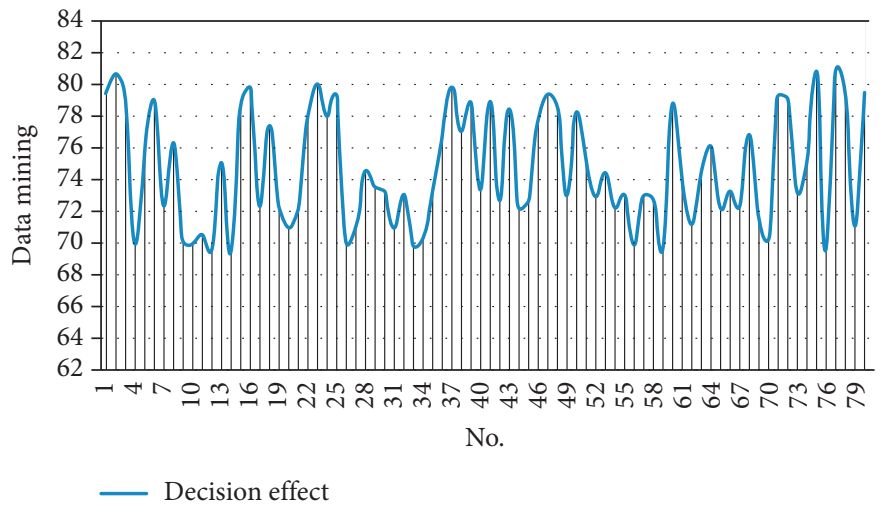

FIgURE 8: Statistical diagram of the evaluation of system decision-making ability.

It can be concluded that the proposed data mining system has more significant potential in deciding while analyzing the development of international agricultural trade.

\section{Conclusion}

Current research on international agricultural trade primarily focuses on subjective comments and environmental impacts. This paper used the bidirectional encoder representations form transformers (BERT) model and constructed a trade data analysis system with efficient data analysis capabilities to increase the evaluation of international agricultural trade development. We conducted mining and analysis on the massive data of international agricultural trade. As a result, we obtained adequate information to make effective decisions. Our results indicate that our model does reasonably well and obtains adequate information from international agricultural trade data. Furthermore, this paper proves the feasibility of the proposed model through experimental research.

\section{Data Availability}

The data used to support the findings of this study are available from the corresponding author upon request.

\section{Conflicts of Interest}

The author declares that there are no conflicts of interest.

\section{References}

[1] C. L. Anderson, T. W. Reynolds, and P. Biscaye, "Economic benefits of empowering women in agriculture: assumptions and evidence," The Journal of Development Studies, vol. 57, no. 2, 2021.

[2] J. Hedlund, S. B. Longo, and R. York, "Agriculture, pesticide use, and economic development: a global examination (1990-2014)," Rural Sociology, vol. 85, no. 2, pp. 519-544, 2020.

[3] J. M. Antle and C. O. Stöckle, "Climate impacts on agriculture: insights from agronomic-economic analysis," Review of Environmental Economics and Policy, vol. 11, no. 2, pp. 299-318, 2017.
[4] S. R. Paramati, N. Apergis, and M. Ummalla, "Dynamics of renewable energy consumption and economic activities across the agriculture, industry, and service sectors: evidence in the perspective of sustainable development," Environmental Science and Pollution Research, vol. 25, no. 2, pp. 1375-1387, 2018.

[5] J. Cai, J. Luo, S. Wang, and S. Yang, "Feature selection in machine learning: a new perspective," Neurocomputing, vol. 300, no. 2, pp. 70-79, 2018.

[6] J. N. Goetz, A. Brenning, H. Petschko, and P. Leopold, "Evaluating machine learning and statistical prediction techniques for landslide susceptibility modeling," Computers \& Geosciences, vol. 81, no. 1, pp. 1-11, 2015.

[7] Y. Xu, F. Jiang, J. Du, and D. Gong, "A cross-domain collaborative filtering algorithm with expanding user and item features via the latent factor space of auxiliary domains," Pattern Recognition, vol. 94, pp. 96-109, 2019.

[8] D. Ramesh and B. Vardhan, "Data mining techniques and applications to agricultural yield data," International Journal of Advanced Research in Computer and Communication Engineering, vol. 2, no. 9, 2013.

[9] M. Motiur Rahman, N. Haq, and R. M. Rahman, "Application of data mining tools for rice yield prediction on clustered regions of Bangladesh," in Proceedings of the 2014 17th International Conference on Computer and Information Technology (ICCIT), pp. 8-13, Dhaka, Bangladesh, December 2014.

[10] K. Verheyen, M. Adrianens, and S. Hermy, "High resolution continuous soil classification using morphological soil profile descriptions," Geoderma, vol. 101, pp. 31-48, 2001.

[11] Y. Xu, Y. Chu, F. Jiang, Y. Guo, and D. Gong, "SVMs classification based two-side cross domain collaborative filtering by inferring intrinsic user and item features," KnowledgeBased Systems, vol. 141, pp. 80-91, 2018.

[12] G. Alberto, F. Juan, and W. Ojeda-Bustamante, "Predictive ability of machine learning methods for massive crop yield prediction," Spanish Journal of Agricultural Research, vol. 12, no. 2, pp. 313-328, 2014.

[13] A. Mouazen, X. Pantazi, D. Moshou, and T. Alexandridis, "Wheat yield prediction using machine learning and advanced sensing techniques," Computers and Electronics in Agriculture, vol. 121, pp. 57-65, 2016.

[14] D. Sundiman, C. H. Wu, A. Mursidi, S. B. P. Johan, and A. Indahingwati, "Knowledge management key factors: an empirical research on small and medium-sized enterprises in Indonesia," International Journal of Business and Systems Research, vol. 13, no. 2, pp. 139-161, 2019. 
[15] K. Anderson, "Agricultural price and trade policy reform in developing countries since 1960," Applied Economic Perspectives and Policy, vol. 1, no. 2, 2010.

[16] Y. Xu, J. Yang, and Z. Xie, "Training SVMs on a bound vectors set based on fisher projection," Frontiers of Computer Science, vol. 8, no. 5, pp. 793-806, 2014.

[17] A. Karthik, A. Murthy, and V. Devarakonda, "Using the contextual language model BERT for multi-criteria classification of scientific articles," Journal of Biomedical Informatics, vol. 112, Article ID 103578, 2020.

[18] X. Yu, D. Zhan, L. Liu, H. Lv, L. Xu, and J. Du, “A privacypreserving cross-domain healthcare wearables recommendation algorithm based on domain-dependent and domain-independent feature fusion," IEEE Journal of Biomedical and Health Informatics, vol. 11, 2021.

[19] I. Beltagy and K. Arman Cohan, "Scibert: a pretrained language model for scientific text," in Proceedings of the 2019 Conference on Empirical Methods in Natural Language Processing, pp. 3615-3620, Hong Kong, China, November, 2019.

[20] K. Schmidt, I. Aumann, I. Hollander, K. Damm, and J. Matthias, "Applying the Analytic Hierarchy process in healthcare research: a systematic literature review and evaluation of reporting," BMC Medical Informatics and Decision Making, vol. 15, no. 112, 2015.

[21] M. Yu, T. Quan, Q. Peng, X. Yu, and L. Liu, "A model-based collaborate filtering algorithm based on stacked AutoEncoder," Neural Computing and Applications, vol. 6, 2021.

[22] N. Sael, T. Hamim, and F. Benabbou, "Implementation of the analytic hierarchy process for student profile analysis," International Journal of Emerging Technologies in Learning, vol. 14, no. 15, 2019. 